J3eA - Vol. 2 - 2 (2003).

DOI : 10.1051/bib-j3ea:2003002

\title{
SPERA : le Service de Préparation à l'Examen RadioAmateur. Une expérience de e-formation au radioamateurisme et à l'électronique.
}

\author{
P. Kadionik (F4CUQ) *, G. Morizet (F5IBD) **, P. Goold *** et F. Tatin *** \\ (ENSEIRB, Bordeaux) \\ Mis en ligne le 4 février 2003.
}

\begin{abstract}
Résumé
Cet article présente le service en ligne SPERA de préparation à l'examen radioamateur. Ce service web interactif proposé par le radio club F6KQH de l'ENSEIRB (École Nationale Supérieure d'Électronique d'Informatique et de Radiocommunications de Bordeaux) permet à tout futur radioamateur ou passionné d'électronique de se préparer chez lui à la licence radioamateur délivrée par l'Autorité de Régulation des Télécommunications (ART) ou plus simplement à l'électronique.

Mots-clés : radioamateur, e-formation, électronique, web, applet, servlet.
\end{abstract}

(C) EDP Sciences, 2003.

\begin{abstract}
* Patrice Kadionik
Ingénieur ENSEIRB.

Docteur de l'Université Bordeaux I en Instrumentation et Mesures.

Patrice Kadionik a travaillé comme Ingénieur Spécialiste Télécom chez SEMA GROUP TELECOM pendant trois ans avent de rejoindre l'ENSEIRB comme Maître de conférences. Rattaché à l'équipe SACT (Synthèse d'Algorithmes et de Circuits pour les Télécommunications) au laboratoire de micoélectronique IXL, il a particulièrement travaillé sur les circuits numériques de compression vidéo. Il collabore depuis peu avec l'équipe Compose du laboratoire d'informatique Labri sur la mise en place d'une plateforme logicielle de développement de services multimédia. Ses activités d'enseignement concernent l'informatique, l'informatique industrielle et les réseaux. Il est radioamateur et co-responsable du club radioamateur F6KQH de l'ENSEIRB.
\end{abstract}

Adresse postale : ENSEIRB, Avenue du Dr Albert Schweitzer, BP 99, F-33402 Talence Cedex, France. e-mail : kadionik@enseirb.fr site web : http://www.enseirb.fr

\section{** Guy Morizet}

Ingénieur ENSERG.

Professeur agrégé de génie électrique.

Guy Morizet est Professeur Agrégé (PRAG) à l'ENSEIRB depuis 1991. Ses activités d'enseignement concernent l'électronique de base, l'électronique radiofréquence et les systèmes numériques. Il est radioamateur et responsable du club radioamateur F6KQH de l'ENSEIRB.

\section{*** Philippe Goold et Frédéric Tatin \\ Ingénieurs ENSEIRB, spécialité électronique.}

Philippe Goold et Frédéric Tatin sont deux anciens élèves ingénieurs de l'ENSEIRB qui ont participé à la mise en place de SPERA dans le cadre de leur projet de fin d'étude. Ils sont tous deux ingénieurs de l'ENSEIRB, promotion 1999. 


\section{Introduction}

Les radioamateurs sont environ 24000 en France et plusieurs millions dans le monde principalement aux ÉtatsUnis. Reconnus par l'Autorité de Régulation des Télécommunications (ART), ils ont passé un examen technique devant les autorités compétentes prouvant leurs compétences techniques et la connaissance de la réglementation radioamateur leur donnant ainsi le droit d'émettre dans les bandes HF (3-30 MHz), VHF (144 MHz), UHF (430 $\mathrm{MHz}$ ) et au-delà. Les radioamateurs sont à l'origine de bien des progrès en radioélectricité (dans la conception d'antennes notamment) et des radiocommunications. Reconnus pour leur compétence et leur dévouement, ils ont maintes fois prouvé leur aptitude à alerter les autorités de la présence de navires en perdition par exemple, à servir de relais en cas de catastrophes naturelles... En France, ils peuvent d'ailleurs être réquisitionnés en cas de plan ORSEC. Contrairement à la $\mathrm{CB}$ où l'accès est libre à tous, l'activité de radioamateurisme est fortement contrôlée par les autorités par le passage d'un examen donnant droit à la licence puis par le contrôle de la teneur des communications. Pour permettre un entraînement dans les conditions réelles de l'examen, le service SPERA de l'ENSEIRB a été créé.

\section{Présentation générale de SPERA}

SPERA, le service de Préparation à l'Examen RadioAmateur est un service web interactif mis en place depuis un an permettant un entraînement dans les conditions réelles de l'examen. Il est accessible depuis n'importe quel navigateur à l'adresse www.enseirb.fr/f6kqh/spera.html. SPERA est basé sur le programme de l'examen exposé dans le « guide du radioamateur » édité par l'ART. SPERA offre deux possibilités d'entraînement : la première en temps limité dans les conditions réelles d'examen, la seconde hors temps avec possibilité de choisir ses questions parmi la réglementation, la technique... En ne choisissant qu'un questionnaire sur la technique, on peut alors se former à l'électronique de base. Une série de QCM (Questions à Choix Multiples) est proposée. Les figures suivantes (Fig. 1, 2 et 3) présentent l'interface graphique de SPERA. 


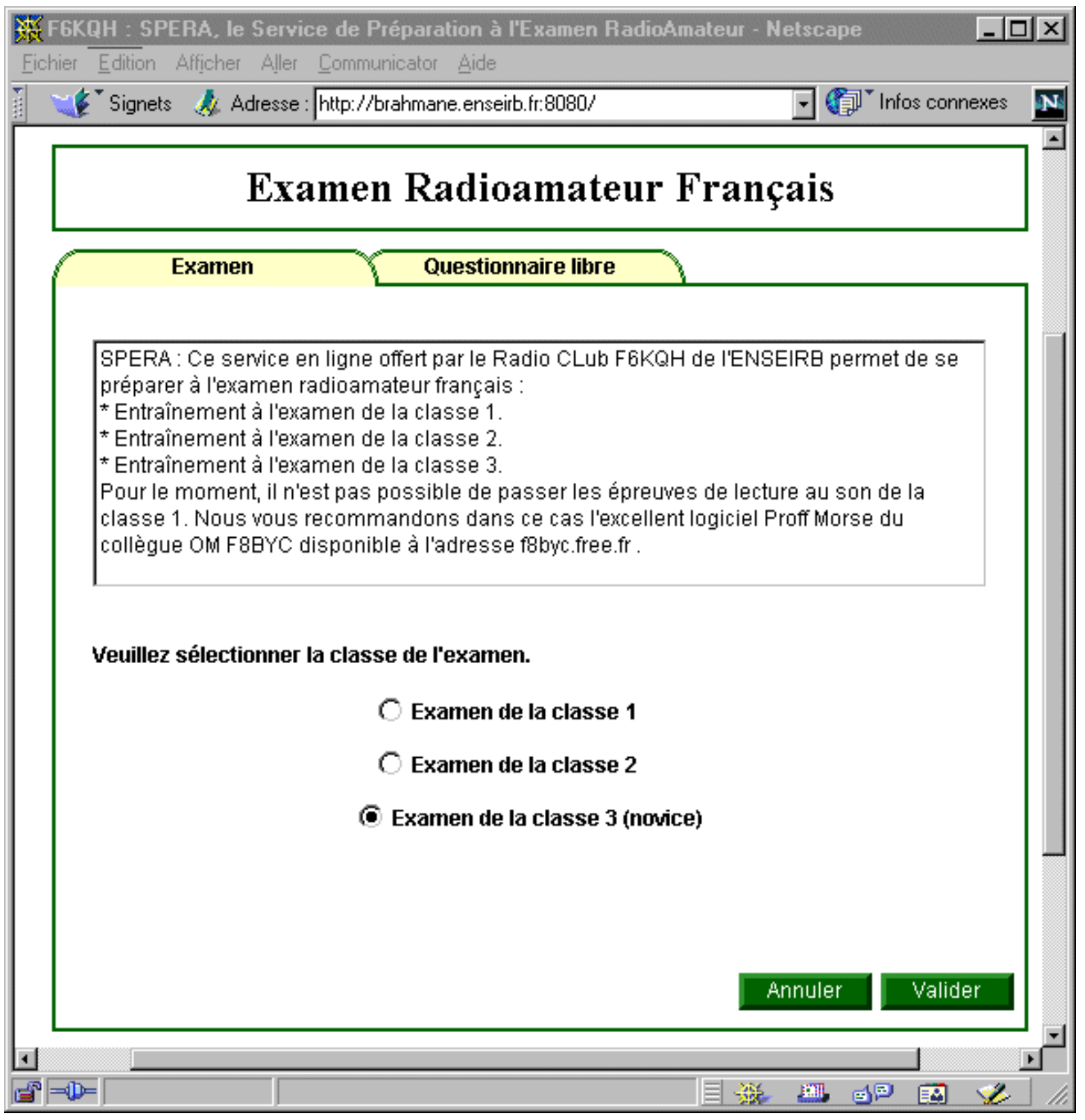

Fig. 1. Interface graphique de SPERA. 


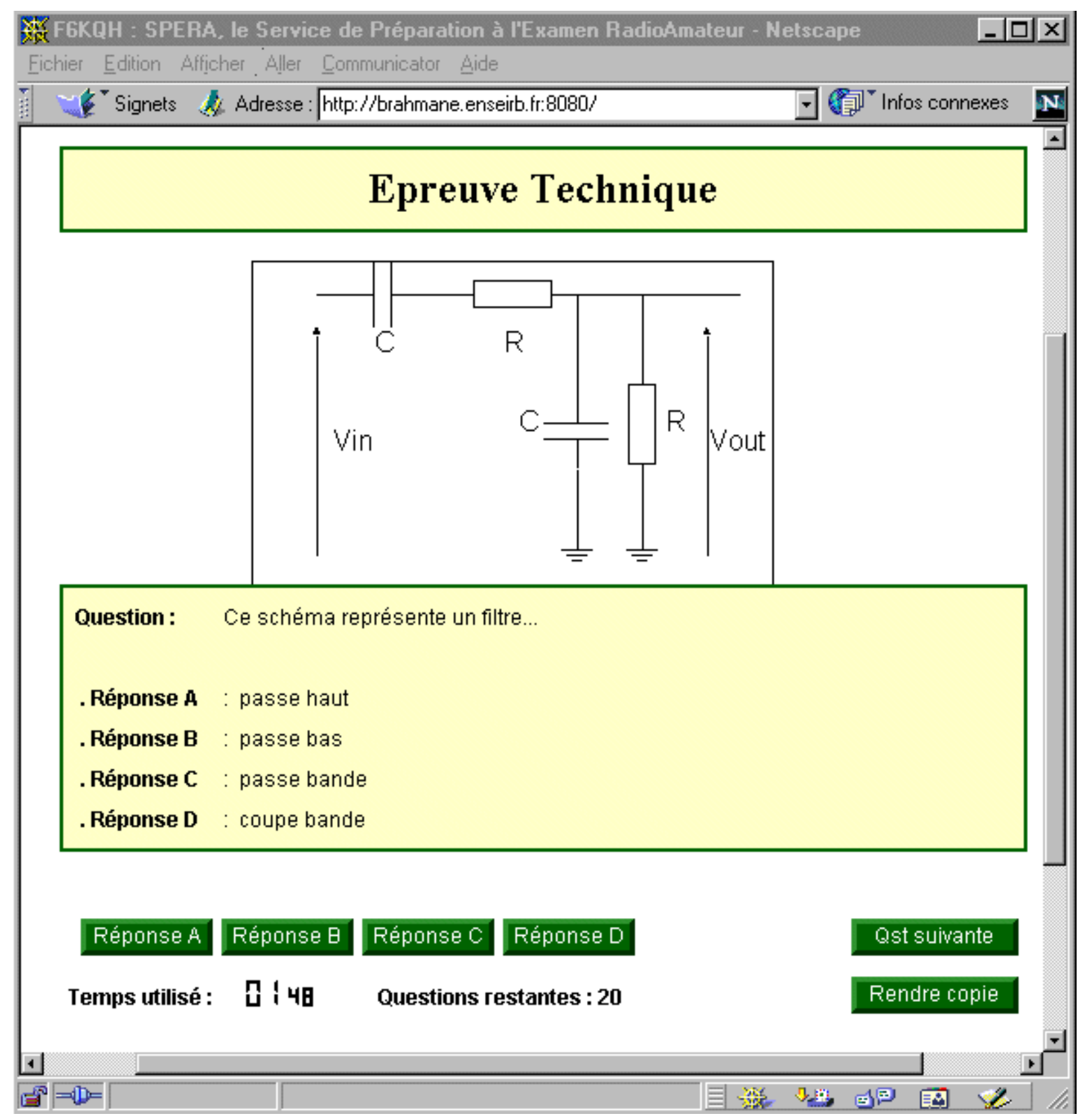

Fig. 2. Question typique de technique de SPERA. 


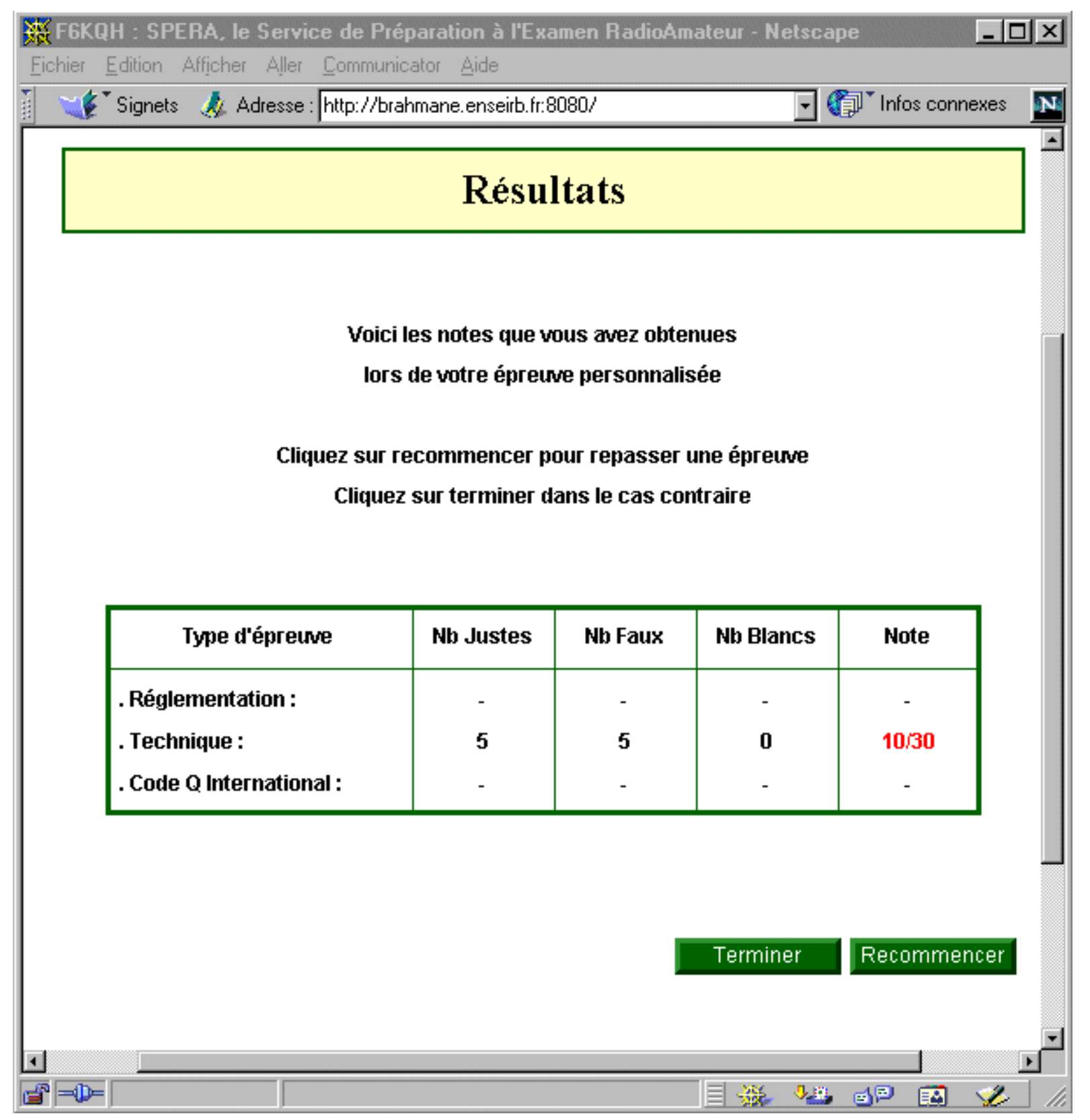

Fig. 3. Résultats de la simulation de l'examen fournis par SPERA.

\section{Implémentation de SPERA}

SPERA met en oeuvre les techniques récentes de l'Internet. Bâtie autour du concept d'application client/serveur [1], SPERA se compose d'une applet Java (côté client) exécutée par le navigateur de l'internaute radioamateur donc l'interface a été présentée sur les figures précédentes. Cette interface graphique reprend l'allure globale de l'application réelle lorsque l'on passe l'examen au centre d'examen. Côté serveur, une servlet Java est mise en oeuvre à travers le module JSERV du serveur web Apache. Cette application serveur a été aussi écrite en langage Java pour assurer une meilleure interactivité comme par exemple dans le cas d'un tirage d'un questionnaire aléatoire.

Les échanges entre le client et de serveur se font à travers la socket de connexion. Il est à noter que l'on échange en fait un objet Java sérialisé contenant les questions tirées au sort par l'application servlet en fonction du type de 
l'examen passé ainsi que les réponses pour limiter les échanges sur Internet. La base de données des questions couvre tous les aspects de l'examen (environ 600 questions d'électronique et de réglementation).

Bien que dédié à l'entraînement au passage de l'examen radioamateur, SPERA peut être facilement adapté à un autre contexte. Les contraintes sont :

- $\quad$ serveur web apache avec module JSERV ;

- quatre questionnaires de type QCM avec au plus quatre choix possibles par question ;

- question sous forme de texte ou d'une image GIF ;

- nombre de questions illimité.

\section{Conclusion}

SPERA est opérationnel officiellement depuis juin 2000. Ce service gratuit est le premier du genre pour les radioamateurs électroniciens et reçoit environ 900 connexions par mois. SPERA est utilisé par les élèves de l'ENSEIRB et la communauté d'internautes désirant se préparer à la licence radioamateur. SPERA ne comptabilise pas les taux d'échec et de réussite. Néanmoins, un retour se fait par email. Il est intéressant de noter que la population des utilisateurs de SPERA est hétérogène : il n'y a pas que des usagers électroniciens. On peut citer le cas d'un pharmacien qui s'est entraîné sur SPERA et qui a ensuite réussi avec brio son examen réel !

SPERA est reconnu et référencé par différents sites radioamateurs et notamment par le REF-Union et a été remarqué par le magazine français Mégahertz (juin 2000).

\section{Références bibliographiques}

[1] P. Kadionik, T. Zimmer, Y. Danto, Instrumentation virtuelle sur le World Wide Web pour faire des mesures réelles. CETSIS 1997. 